\title{
The value of joint ultrasonography in predicting arthritis in seropositive patients with arthralgia: a prospective cohort study
}

Marian H. van Beers-Tas ${ }^{1 * \dagger}$, Annelies B. Blanken ${ }^{1 \dagger}$, Mark M. J. Nielen², Franktien Turkstra', Conny J. van der Laken ${ }^{3}$, Marlies Meursinge Reynders ${ }^{1}$ and Dirkjan van Schaardenburg ${ }^{1,4}$

\begin{abstract}
Background: The value of joint ultrasonography (US) in the prediction of clinical arthritis in individuals at risk of developing rheumatoid arthritis (RA) is still a point of debate, due to varying scanning protocols and different populations. We investigated whether US abnormalities assessed with a standard joint protocol can predict development of arthritis in seropositive patients with arthralgia.

Methods: Anti-citrullinated protein antibodies and/or rheumatoid factor positive patients with arthralgia, but without clinical arthritis were included. US was performed at baseline in 16 joints: bilateral metacarpophalangeal 2-3, proximal interphalangeal 2-3, wrist and metatarsophalangeal (MTP) joints 2-3 and 5. Images were scored semi-quantitatively for synovial thickening and for positive signs on power Doppler (PD). Association between US abnormalities and arthritis development at the joint and at the patient level was evaluated. Also, we investigated the added value of US over clinical parameters.

Results: Out of 163 patients who underwent US examination, 51 (31\%) developed clinical arthritis after a median follow-up time of 12 (interquartile range 5-24) months, of which 44 (86\%) satisfied the 2010 American College of Rheumatology/European League Against Rheumatism classification criteria for RA. US revealed synovial thickening and PD in at least one joint in 49 patients (30\%) and 7 patients (4\%), respectively. Synovial thickening was associated with both development and timing of clinical arthritis in any joint (patient level) when MTP joints were excluded from the US assessment (odds ratio 6.6, confidence interval (CI) 1.9-22), and hazard ratio 3.4, Cl 1.6-6.8, respectively, with a mean time to arthritis of 23 versus 45 months when synovial thickening was present versus not present). There was no association between US and arthritis development at the joint level. Predictive capacity was highest in the groups with an intermediate and high risk of developing arthritis based on a prediction rule with clinical parameters.

Conclusions: Synovial thickening on US predicted clinical arthritis development at the patient level in seropositive patients with arthralgia when MTPs were excluded from the US assessment. Positive PD signs were infrequently seen in these at-risk individuals and was not predictive. In patients at intermediate risk of RA, US may help to identify those at higher risk of developing arthritis.
\end{abstract}

Keywords: Ultrasonography, Synovial thickness, Power Doppler, Arthralgia patients, Arthritis, Seropositive, Autoantibodies

\footnotetext{
* Correspondence: m.v.beers@reade.nl

${ }^{\dagger}$ Marian H. van Beers-Tas and Annelies B. Blanken contributed equally to this work.

${ }^{1}$ Amsterdam Rheumatology and immunology Center | Reade, Dr. Jan van

Breemenstraat 2, 1056 AB Amsterdam, The Netherlands

Full list of author information is available at the end of the article
}

(c) The Author(s). 2018 Open Access This article is distributed under the terms of the Creative Commons Attribution 4.0 International License (http://creativecommons.org/licenses/by/4.0/), which permits unrestricted use, distribution, and reproduction in any medium, provided you give appropriate credit to the original author(s) and the source, provide a link to the Creative Commons license, and indicate if changes were made. The Creative Commons Public Domain Dedication waiver (http://creativecommons.org/publicdomain/zero/1.0/) applies to the data made available in this article, unless otherwise stated. 


\section{Background}

Rheumatoid arthritis (RA) is a chronic autoimmune disease that is characterized by synovial inflammation and swelling. In the at-risk phase before clinical RA development, the presence of autoantibodies such as anti-citrullinated protein antibodies (ACPA) and/or IgM-rheumatoid factor (RF) with or without arthralgia symptoms predict the development of RA [1-4]. Early treatment of RA improves the outcome [5], and this principle may also apply to the preclinical phase of RA. Detecting patients with arthralgia at high risk of RA offers the opportunity to develop treatment strategies for prevention of RA in these patients. Current prediction rules for arthritis development based on clinical parameters (including autoantibodies) are suitable for this $[4,6-8]$, but their predictive value seems too low to ensure that all patients would be treated validly with medication with potentially serious side effects. The predictive capacity might be substantially improved by adding imaging [9].

Ultrasonography (US) is widely available at relatively low cost and has no radiation exposure. There is evidence that US increases diagnostic certainty when compared to clinical examination alone for diagnosing RA in early undifferentiated arthritis [10-15]. US was also described to add value to clinical examination in individuals at risk of developing RA $[9,11,16-20]$, which may be particularly the case for power Doppler (PD) abnormalities $[9,11$, $19]$ and mainly in autoantibody-negative persons [17, 18]. However, discrepancies related to the definition of US synovial thickness [21], the selection of joints included in the US protocol [21] and the use of different scoring systems [22-25] hamper general clinical implementation of US to help diagnose and predict RA [26].

In a previous study on the value of US in the prediction of arthritis in seropositive patients with arthralgia, we only scanned painful and adjacent/contralateral joints (which differed between patients) and showed that arthritis could be predicted at the joint but not at the patient level [16]. The present follow-up study included a new cohort of seropositive patients with arthralgia, in which we investigated the value of an US protocol including a standardized set of joints (regardless of local clinical symptoms) to predict clinical arthritis development. We also evaluated whether US abnormalities add predictive value to clinical parameters.

\section{Methods}

\section{Study population}

Seropositive patients with arthralgia (ACPA and/or RF), but without clinical arthritis, were recruited at Reade (Amsterdam) between March 2009 and December 2015. At the start of the study patients had very recently (0-2 weeks preceding inclusion) been evaluated by the treating rheumatologist who concluded they had arthralgia with autoantibodies and referred them to participate in the study. All had a new evaluation at study baseline where the study physician, who was trained in performing joint counts, collected data. In case of doubt about the presence of arthritis, another rheumatologist (involved in the study group) also performed the joint exam at this visit and made the final decision on presence or absence of arthritis. Patients with past arthritis or arthritis at baseline (defined as one or more swollen joints as reported by two independent investigators), age $<18$ years and $>70$ years, previous treatment with a disease-modifying antirheumatic drug or recent glucocorticoid treatment, systemic autoimmune disease, systemic infections, lymphoproliferative disorders or recent radiotherapy were excluded from the cohort $[2,16]$. Medical history, tender joint count in 53 joints (TJC53), details of joint symptoms and ACPA/RF status were recorded at baseline [2], together with clinical criteria included in a previously described prediction rule for the development of arthritis in seropositive patients with arthralgia: presence of a first-degree relative with RA, alcohol consumption, symptom onset $<12$ months, presence of intermittent symptoms, presence of symptoms in upper and lower extremities, presence of joint swelling (anamnestic), visual analog scale assessing pain $(\geq 50 \mathrm{~mm})$ and morning stiffness lasting at least $1 \mathrm{~h}$ [4]. These parameters (combined with the autoantibody status) were used to calculate a risk rule score ranging from 1 to 13 , divided into three risk groups (low 0-4, intermediate 5-6, high 7-13). During yearly follow up, for up to 5 years, clinical arthritis development in any of 44 joints was assessed by a trained physician and an extra visit could be scheduled when arthritis development was suspected. If clinical arthritis was present in at least one joint, this was confirmed by a senior rheumatologist (DvS) without knowledge of the patient's serostatus. The study was approved by the Slotervaart ziekenhuis and Reade ethics committee. Signed informed consent was obtained from all patients prior to inclusion.

\section{Ultrasonography}

The joints were scanned according to a predefined standard US protocol of those 16 joints in which clinical swelling had developed most often in our previous US pre-RA cohort: bilateral wrists, metacarpophalangeal (MCP) 2-3, proximal interphalangeal (PIP) 2-3 and metatarsophalangeal (MTP) 2-3 and 5 [16]. All scans were performed using the Acuson Antares ultrasound system, premium edition (Siemens, Malvern, PA, USA) using linear array transducers VF 13-5 SP for

finger and toe joints (operating at $11.43 \mathrm{MHz}$ for grayscale and $8.9 \mathrm{MHz}$ for PD) and VF 13-5 for larger joints (operating at $11.43 \mathrm{MHz}$ for grayscale and 7.3 $\mathrm{MHz}$ for PD), according to the manufacturer's criteria [16]. The joints were scanned in the dorsal longitudinal plane from the most lateral to the most medial site and in the transverse plane 
from the proximal to distal site of the joint. Finger joints were also scanned in the palmar longitudinal plane. The wrist included scans of the radiocarpal and intercarpal joints and ulnocarpal joint including the ulnar styloid process. Effusion and synovial hypertrophy were scored in a combined measure (synovial thickening) as both phenomena often appear concurrently [27]. Synovial thickening and PD signs were scored using the four-grade semi-quantitative scale (0-3) of Szkudlarek [16, 22]. Synovial thickening grade $\geq 2$ and $P D$ grade $\geq 1$ were regarded as abnormal. When multiple images were made of one joint, the highest score was used to obtain a single score per joint. US examinations were all performed by a single radiologist (MMR) experienced in musculoskeletal US, who was blinded to the clinical data. Ultrasound results were not available to the treating physician nor the physician performing follow-up study visits and thus the abnormalities did not change the way study participants were evaluated. Data were analyzed after all data were collected.

\section{Statistics}

Normally distributed continuous data were summarized by the mean and standard deviation (SD). Non-normally distributed data were summarized by the median and interquartile range (IQR). The risk of arthritis development at the patient level was estimated by the chi-square or Fisher's exact test, and corresponding positive and negative predictive values (PPV, NPV) were calculated. Results were expressed as odds ratios (OR) with 95\% confidence interval (CI). Timing of arthritis development was assessed by Kaplan-Meier survival analysis using the log-rank test and Cox regression analysis, expressed as mean time to arthritis (we reported mean survival time instead of the mostly preferred median survival time, because in order to calculate the median, $50 \%$ of subjects need to develop arthritis and this was not the case in any of our groups) and hazard ratios (HR) with 95\% CI. We also performed multivariate regression analysis to look at the additional value of US over clinical parameters in the patients with low, intermediate or high risk of developing RA [4]. Subgroup analyses were performed in ACPA-positive versus ACPA-negative patients. All analyses at the patient level were performed with and without inclusion of the MTP joints, as a previous study indicated that the frequency of synovial thickening in the MTP joints in healthy controls was too high to discriminate between those who will develop arthritis and those who will not [19]. The risk of arthritis development at the joint level (using all joints) was analyzed using generalized estimating equations (GEE) with an exchangeable correlation matrix, allowing correction for within-patient correlation [28]. Statistical analysis was performed using SPSS version 22 statistics software (SPSS Inc., Chicago, IL, USA).

\section{Results}

In total, 287 seropositive patients with arthralgia were consecutively screened through our prospective cohort in the inclusion period. There were 14 patients excluded due to clinical arthritis at baseline, 99 patients did not receive US examination due to logistical problems or not consenting to US and 11 patients were lost to follow up after their baseline measurement. The remaining 163 patients were analyzed in the current study ( $74 \%$ female, mean \pm SD) age $51 \pm 11$ years). Their baseline characteristics are shown in Table 1. Baseline characteristics of those who were included were similar to those who were not (data not shown). There were 51 patients (31\%) who developed clinical arthritis after a median follow up of 12 (IQR 5-24) months: 44 patients (86\%) who developed arthritis satisfied the 2010 American College of Rheumatology (ACR)/European League Against Rheumatism (EULAR) classification criteria for RA. The 112 patients who did not develop arthritis had a median follow-up time of 28 (IQR 19-49) months. US was performed within a median of 3 weeks (IQR 2-6 weeks) after the first visit. If we look at the distribution of pain among the 53 joints at baseline, we see that the shoulder was most often painful, followed by MTP3, acromioclavicular joint, sternoclavicular joint, MTP4, MTP2, wrist and knee. At the time of arthritis development, 6 out of 8 bilateral joints that were included in the ultrasound protocol (wrist, MCP2, PIP2, PIP3, MTP2 and MTP3) were among the 8 joints that were most often painful (together with shoulder and MTP4). Data on severity of pain were not available. There was no statistically significant association between pain and synovial thickening at baseline using GEE analysis (OR 2.1, CI 0.9-4.7, $p=0.08$ ). When we excluded the MTPs from analysis we found association between pain and synovial thickening corrected for within-patient correlation (OR 3.5, CI 1.1-11.4, $p<0.05$ ).

Table 1 Baseline characteristics

\begin{tabular}{ll}
\hline Baseline characteristics & $\begin{array}{l}\text { Value in study } \\
\text { population } \\
(n=163)\end{array}$ \\
\hline Age in years, mean \pm SD & $51 \pm 11$ \\
Female sex, $n(\%)$ & $121(74 \%)$ \\
Arthralgia duration in months, median (IQR) & $13(6-36)$ \\
Number of reported painful joints, median (IQR) & $8(4-19)$ \\
Tender joint count (53 joints), median (IQR) & $1(0-5)$ \\
VAS pain in mm (0-100), mean \pm SD & $35 \pm 25$ \\
Antibody status & \\
ACPA negative, RF positive, $n(\%)$ & $72(44 \%)$ \\
ACPA positive, RF negative, $n(\%)$ & $44(27 \%)$ \\
ACPA positive, RF positive, $n(\%)$ & $47(29 \%)$ \\
\hline
\end{tabular}

$A C P A$ anti-citrullinated protein antibodies, IQR interquartile range, $R F$ rheumatoid factor, $S D$ standard deviation, VAS visual analog scale 
US and clinical arthritis development at the patient level At baseline, 49 patients (30\%) had US synovial thickening and 7 patients (4\%) had PD abnormalities in at least one joint (Table 2): of these, 5 patients (3\%) had both synovial thickening and PD abnormalities in at least one joint and 3 patients (2\%) had both synovial thickening and PD abnormalities in the same joint (with 1 patient having 4 joints with both synovial thickening and PD abnormalities). When excluding the MTP joints, 14 patients (9\%) had synovial thickening in at least one joint and 7 patients (4\%) had PD abnormalities.

Of the patients with US abnormalities in at least one joint, the median number of affected joints with synovial thickening was 2 (min-max 1-6) in the patients developing arthritis and also 2 (min-max 1-4) in the patients who did not develop arthritis. For PD abnormalities these numbers were 1 (1-5) and $3(1-3)$, respectively.

A greater proportion of patients with US synovial thickening at baseline in at least one joint developed arthritis although this was not statistically significant (Table 2). This trend appeared to be more pronounced and was significant when the MTP joints were excluded (OR 6.6, CI 1.9-22.2, $p<0.01$ ). The corresponding positive predictive value (PPV) and negative predictive value (NPV) were $71 \%$ and $72 \%$, respectively. There was no statistically significant association between the presence of PD abnormalities in one or more joints and clinical arthritis development (OR 0.9, CI 0.1-4.7, $p=1.0$, PPV $29 \%$, NPV 69\%). All patients with PD abnormalities in the MTP joints also had PD abnormalities in at least one other joint, therefore the association did not change when the MTP joints were excluded. Sensitivity analysis, with the development of RA defined according to the ACR/EULAR 2010 classification criteria as the outcome, showed a slight increase in the odds ratios and predictive values as compared to clinical arthritis as the outcome
(OR 8.5, CI 2.4-28.7, $p=<0.01$, PPV 71\%, NPV 77\%; Table 2).

Clinical arthritis developed earlier in patients who had US synovial thickening in at least one joint than in those without US synovial thickening, but this was only the case when the MTP joints were excluded from the US assessment (mean time to arthritis 23 versus 45 months, $p<0.01$; Fig. 1). The corresponding HR was 3.4 (CI 1.6-6.7, $p<0.01$ ). Patients with PD abnormalities (both with and without the MTP joints included) did not develop arthritis earlier than patients without PD abnormalities (mean time to arthritis 44 versus 43 months, $p=0.7$; Fig. 1 ) and the corresponding HR of 0.8 (CI $0.1-3.2, p=0.7$ ) was not statistically significant. In Fig. $1 \mathrm{~b}$ the lines cross due to small numbers of PD-positive patients. Since this could be caused by effect modification, we investigated whether there was a significant difference between the effects in patients before and after a cutoff value of 30 months, but this was not the case.

We did not demonstrate clinically relevant differences in US abnormalities for prediction of arthritis at the patient level between ACPA-positive and ACPA-negative patients, except for synovial thickening without the MTP joints included in the ACPA-positive patients (19\% developed arthritis versus $2 \%$ who did not; note the small numbers and thus wide CIs; Additional file 1: Table S1). The same was true for ACPA-positive versus ACPA-positive and RFpositive patients (Additional file 2: Table S2).

\section{US and clinical arthritis development at the joint level}

US was performed at baseline on 2608 joints (16 joints per patient, no missing data). US revealed synovial thickening in $105 / 2608$ (4\%) of the joints, mostly in the MTP joints $(84 / 105(80 \%))$. PD abnormality was seen in 14/2608 $(0.5 \%)$ of the joints (of which $3 / 14$ (21\%) were in the MTP joints). In $158 / 2608$ (6\%) of the scanned joints at baseline,

Table 2 Association between ultrasound abnormalities and development of clinical arthritis/rheumatoid arthritis analyzed at the patient level

\begin{tabular}{|c|c|c|c|c|c|c|}
\hline Ultrasound abnormalities & Arthritis, yes & Arthritis, no & OR $(95 \% \mathrm{Cl})$ & $p$ value & PPV & NPV \\
\hline Outcome: clinical arthritis & $n=51$ & $n=112$ & & & & \\
\hline Synovial thickening ${ }^{a}$ (16 joints) & $19(37 \%)$ & $30(27 \%)$ & $1.6(0.8-3.3)$ & $0.18^{\mathrm{c}}$ & $39 \%$ & $72 \%$ \\
\hline Synovial thickening (10 joints, no MTP) & 10 (20\%) & $4(4 \%)$ & $6.6(1.9-22.2)$ & $<0.01^{d}$ & $71 \%$ & $72 \%$ \\
\hline Power Doppler ${ }^{a}\left(16\right.$ joints) ${ }^{b}$ & $2(4 \%)$ & $5(5 \%)$ & $0.9(0.1-4.7)$ & 1.0. ${ }^{d}$ & $29 \%$ & $69 \%$ \\
\hline Outcome: 2010 RA criteria & $n=44$ & $n=119$ & & & & \\
\hline Synovial thickening (16 joints) & 17 (39\%) & $32(27 \%)$ & $1.7(0.8-3.6)$ & $0.15^{c}$ & $35 \%$ & $76 \%$ \\
\hline Synovial thickening (10 joints, no MTP) & $10(23 \%)$ & $4(3 \%)$ & $8.5(2.4-28.7)$ & $<0.01^{d}$ & $71 \%$ & $77 \%$ \\
\hline Power Doppler (16 joints) ${ }^{b}$ & $2(5 \%)$ & $5(4 \%)$ & $1.1(0.2-5.9)$ & $1.0^{\mathrm{d}}$ & $29 \%$ & $73 \%$ \\
\hline
\end{tabular}

Cl confidence interval, MTP metatarsophalangeal, NPV negative predictive value, $O R$ odds ratio, $P P V$ positive predictive value, $R A$ rheumatoid arthritis ${ }^{a}$ Results are presented for synovial thickening and power Doppler in at least one joint

${ }^{b}$ Same results when excluding MTP joints

${ }^{c}$ Chi-square test

${ }^{\mathrm{d}}$ Fisher's exact test 

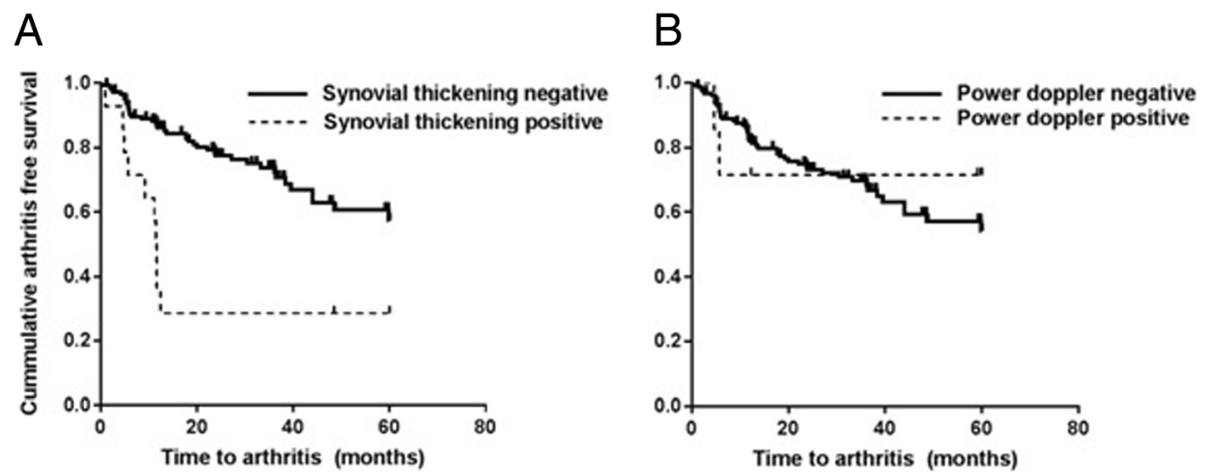

Fig. 1 Kaplan-Meier curves for synovial thickening (a) and power Doppler (b) and time to arthritis development (months). The metatarsophalangeal joints were excluded

clinical arthritis developed during follow up (32\% of this in MTP joints). At that time, the median number of joints with arthritis per patient was 3 (range $0-13$, note that arthritis developed outside the standard set of 16 joints in 5 of the patients). Of the 158 joints with arthritis, US evidence of synovial thickening was only seen in the same joint at baseline in 8 joints (5.1\%) (for PD this was 1 joint $(0.6 \%))$. There was no statistically significant association between the presence of synovial thickening in a joint and development of arthritis development in the same joint (OR 1.0, CI 0.3-2.9, $p=1.0$ ) or between the presence of PD abnormality and development of arthritis in the same joint (OR 1.0, CI 0.2-4.8, $p=1.0$ ) using GEE analysis.

\section{Added value of US for prediction of clinical arthritis versus clinical parameters alone}

Patients were divided into three groups of low, intermediate and high risk of developing arthritis according to the clinical prediction rule score described in "Methods". Multivariate regression analysis including the clinical prediction rule risk groups and synovial thickening in all joints excluding the MTP joints produced an OR of 6.1 (CI 1.6-23.2, $p<0.01$ ) for synovial thickening and 3.5 (CI 2.2-5.5, $p<0.01$ ) for the prediction rule groups. The number of patients in each group was too small to perform proper subgroup analysis, however, the relatively high OR of 6.1 for risk of synovial thickening seems to be due to both the patients from the intermediate risk group (in which four patients with synovial thickening developed arthritis and only one did not) and the high risk group (in which all six patients with synovial thickness also developed arthritis, see Table 3). For PD the OR in multivariate regression was $1.7(0.3-10.2, p=0.55)$, with an OR of $3.6(2.3-5.6, p<0.01)$ for the clinical score.

\section{Discussion}

Here we investigated whether abnormalities found with a standardized US protocol are useful in the prediction of arthritis development in seropositive patients with arthralgia, and whether these US abnormalities add predictive value over clinically available parameters. Synovial thickening on US (wrist and hand joints, excluding the MTPs) was associated with both arthritis development and its timing, at the patient level but not at the joint level. Also, US synovial thickening in the wrist and hand joints adds predictive value in patients with an intermediate-to-high risk of developing arthritis based on a clinical prediction rule. PD abnormalities on US were not associated with arthritis development.

The results should be interpreted in the light of small numbers of patients with US abnormalities, especially for PD. There were $31 \%$ of patients with abnormalities on US in at least one joint, which decreased to $10 \%$ when not analyzing the MTP joints. In total, only $4 \%$ presented with PD. Therefore, even in our population with a relatively high risk of developing arthritis (around 30\%), a large number needs to be screened to find only a small proportion of patients with US abnormalities that progress to arthritis. This is undesirable for clinical implementation of US.

The usefulness of US as a predictor of arthritis or RA development has been described by several authors with varying results $[9,11,16,18,19]$. Our previous report of evaluating only painful joints concluded that presence of US abnormalities (both synovial thickness and PD) was associated with arthritis development at the joint level, but not at the patient level, which is opposite to our current conclusion [16]. A group from Leeds performed a study that included 136 ACPA-positive patients with musculoskeletal symptoms and showed that synovial thickening in two or more joints was related to 2.3 times greater chance of developing arthritis at the patient level, which increased to 3.7 for PD in at least 2 joints [19]. The HRs were even higher when analyzing on joint level (HR 9.4 for synovial thickening score $\geq 2$ in a joint and HR 31 for $P D \geq 2$ ). In another study, the same group found that PD signal added predictive value to clinical parameters in the prediction of arthritis [9] The higher scores (namely for PD) compared to our study may have been caused by the selection of patients with a higher a priori risk, as they were all ACPA-positive patients with 
Table 3 Added value of ultrasound over clinical parameters according to a clinical prediction rule

\begin{tabular}{|c|c|c|c|c|}
\hline Ultrasound abnormalities & Arthritis, yes & Arthritis, no & OR $(95 \% \mathrm{Cl})^{\mathrm{d}}$ & $p$ value \\
\hline Clinical prediction rule risk-groups $^{a}$ & $n=51$ & $n=112$ & & \\
\hline Synovial thickening ${ }^{\mathrm{b}}$ (16 joints) & & & $1.5(0.7-3.4)$ & 0.3 \\
\hline Clinical prediction rule & & & $3.5(2.2-5.4)$ & $<0.01$ \\
\hline Low risk & $2 / 8(25 \%)$ & 19/67 (28\%) & & \\
\hline Intermediate risk & $5 / 13(38 \%)$ & $5 / 25(20 \%)$ & & \\
\hline High risk & $12 / 30(40 \%)$ & $6 / 20(30 \%)$ & & \\
\hline Synovial thickening (10 joints, no MTP) & & & $6.1(\mathrm{Cl} 1.6-23.2)$ & $<0.01$ \\
\hline Clinical prediction rule & & & $3.5(\mathrm{Cl} 2.2-5.5)$ & $<0.01$ \\
\hline Low risk & $0 / 8(0 \%)$ & $3 / 67(4 \%)$ & & \\
\hline Intermediate risk & $4 / 13(31 \%)$ & $1 / 25(4 \%)$ & & \\
\hline High risk & $6 / 30(20 \%)$ & $0 / 20(0 \%)$ & & \\
\hline Power Doppler ${ }^{b}\left(16\right.$ joints) ${ }^{c}$ & & & $1.7(0.3-10.2)$ & 0.5 \\
\hline Clinical prediction rule & & & $3.6(2.3-5.6)$ & $<0.01$ \\
\hline Low risk & $0 / 8(0 \%)$ & $4 / 67(6 \%)$ & & \\
\hline Intermediate risk & $2 / 13(15 \%)$ & $1 / 25(4 \%)$ & & \\
\hline High risk & $0 / 30(0 \%)$ & $0 / 20(0 \%)$ & & \\
\hline
\end{tabular}

$A C P A$ anti-citrullinated protein antibody, $C l$ confidence interval, MTP metatarsophalangeal, OR odds ratio, $R F$ rheumatoid factor

${ }^{\text {a }}$ Risk groups based on the clinical prediction rule described in reference number 4

${ }^{\mathrm{b}}$ Results are presented for synovial thickening and power Doppler in at least one joint (present, \%)

'Same results when excluding MTP joints

${ }^{\mathrm{d}}$ Logistic regression analysis (note that the prediction rule risk groups were combined)

arthralgia. A study from Switzerland focused on very early arthritis and evaluated 49 patients with inflammatory hand symptoms of recent onset ( $\leq 12$ weeks) with or without clinical arthritis. Since all ACPA-positive and/or RF-positive patients eventually developed arthritis, the value of US was only determined in the seronegative patients [18]. In this subgroup the post-test probability in patients with 1-3 clinical parameters could be raised from $2-30 \%$ to $50-94 \%$ when using US synovial thickness or PD abnormality. Finally, synovial thickness (PD was not analyzed) was also researched in another seronegative patient population of 80 , in which the OR of arthritis and/ or RA development was 7.5 (clinical parameters were not taken into account) [18].

Three main reasons may have caused the different results presented above. First, different US scanning protocols were used. Our study in combination with our previous study indicates that applying a US protocol with a standardized set of joints results in better prediction at the patient level and that scanning only painful joints results in better prediction at the joint level. Although it appears attractive to scan more joints, both symptomatic and asymptomatic, this will make US more time-consuming and thus unfit for a clinical setting [29]. Also, it is still hard to define which set of asymptomatic joints should be used. In order to improve the selection of joints in the US protocol, future studies to determine in which joints ultrasound abnormalities are most predictive of arthritis development are needed. However, our study and a previous one has shown that it may be useful to exclude the MTP joints [19]. This would be convenient since it reduces the time taken to perform the US examination. The second reason for different results is technical differences between US machines, which mainly seems to be important for detection of the PD signal [30]. It is possible that in future studies, discrimination between effusion and synovial hypertrophy of the MTP joints may enhance prediction of those developing arthritis [31]; however, based on the available literature before the start of the study, effusion and synovial hypertrophy were believed to be part of the same pathophysiological process and were often seen combined, which was the reason for scoring them as one in the current study [27]. Differences between groups may be overcome in the future as the availability of good-quality US machines increases. The final reason is the fact that the use of US in prediction depends highly on the a priori chance of developing arthritis in the population that is investigated. In patients with an already high risk, for instance those who are ACPA-positive (for example, 42\% developed arthritis in the Leeds cohort [19], 46\% in the present study) or those with a high probability based on clinical prediction rules, having US abnormalities was almost always associated with arthritis development (6 patients in the present study, with a $100 \%$ chance of developing arthritis) $[9,17,19]$. However, US might be of even more value in those subpopulations of at-risk patients in which there is 
more diagnostic uncertainty, such as in seronegative patients with arthralgia $[17,18]$ and patients scoring intermediate on the clinical prediction rule. We did not include seronegative patients, but we did show that among the 5 patients with an intermediate risk of developing arthritis, $80 \%$ had US abnormalities.

Some additional comments can be made. First, it may be interesting to look not only at US abnormalities, but also to the absence of these in relation to a lower chance of developing arthritis. Van der Ven et al. [32], reported NPV of $89 \%$ for grayscale and/or PD abnormalities in 196 patients with inflammatory arthralgia. In the present study, the NPVs were somewhat lower for synovial thickness (72\%) and PD abnormalities (77\%) individually, although they were measured in a cohort with a low prevalence of US abnormalities. Second, it may be worthwhile to include tenosynovitis as an independent variable besides synovitis when looking at US abnormalities in the at-risk phase of RA [33]. Third, it was speculated that US is of greater value when applying the 2010 criteria for RA, because these criteria are designed to identify RA at an early stage [11]. This was confirmed by our study as both OR and NPV increased when the 2010 criteria for RA were used as the outcome measure. Last, a limitation of this study may be that US examinations were all performed by a single radiologist, although this radiologist had a high interobserver agreement between this radiologist and another was high in our previous study. [16].

\section{Conclusions}

In conclusion, synovial thickening on US using a standard US protocol with exclusion of the MTPs predicted arthritis development and its timing in seropositive patients with arthralgia. PD did not predict arthritis development, probably related to low PD frequency. A large study population needs to be screened to find only a small percentage of patients with US abnormalities, so expected use for routine clinical practice and to select individuals at risk of developing arthritis for preventive studies is low. However, based on our data we do expect that US can be of additional use for clinicians in those patients who have an intermediate risk of developing arthritis when calculating the prediction rule, as compared to those patients for whom the risk is more clearly defined based on clinical parameters (low and high risk).

\section{Additional files}

Additional file 1: Table S1. Association of ultrasound abnormalities with clinical arthritis development, ACPA-positive versus ACPA-negative patients (patient level). (DOCX $14 \mathrm{~kb}$ )

Additional file 2: Table S2. Association of ultrasound abnormalities with clinical arthritis development, only ACPA-positive versus ACPA-positive and RF-positive patients (patient level). (DOCX $15 \mathrm{~kb}$ )

\section{Abbreviations}

ACPA: Anti-citrullinated protein antibodies; Cl: Confidence interval; DvS: Dirkjan van Schaardenburg; GEE: Generalized estimating; HR: Hazard ratio; IQR: Interquartile range; MCP: Metacarpophalangeal; MMR: Marlies Meursinge Reynders; MTP: Metatarsophalangeal; NPV: Negative predictive value; OR: Odds ratios; PD: Power Doppler; PIP: Proximal interphalangeal; PPV: Positive predictive value; RA: Rheumatoid arthritis; RF: Rheumatoid factor; SD: Standard deviation; TJC53: Tender joint count 53;

US: Ultrasonography/ultrasound

\section{Acknowledgements}

We thank Ronald van Volienhoven for critically reviewing the manuscript.

\section{Funding}

This project was partly funded by the Dutch Arthritis Association (8-1-306).

\section{Availability of data and materials}

The datasets used and/or analyzed during the current study are available from the corresponding author on reasonable request.

\section{Authors' contributions}

All authors contributed substantially to the conception and design of the study and interpreted data. FT, CJvdL, MMR and DvS designed the ultrasound protocol. MHVBT and MMR collected data. MHVBT and ABB analyzed data. MHvBT, $A B B, M M J$ and DvS prepared the first draft of the report. All authors contributed to revision of the report and approved the final version.

\section{Ethics approval and consent to participate}

This study was approved by the Slotervaart ethics committee. Signed informed consent was obtained from all patients prior to inclusion.

\section{Consent for publication}

All authors gave their consent for publication.

\section{Competing interests}

The authors declare that they have no competing interests.

\section{Publisher's Note}

Springer Nature remains neutral with regard to jurisdictional claims in published maps and institutional affiliations.

\section{Author details}

${ }^{1}$ Amsterdam Rheumatology and immunology Center | Reade, Dr. Jan van Breemenstraat 2, 1056 AB Amsterdam, The Netherlands. ${ }^{2}$ Netherlands Institute for Health Services Research (NIVEL), Otterstraat 118-124, 3513 CR Utrecht, The Netherlands. ${ }^{3}$ Amsterdam Rheumatology and immunology Center, VU University Medical Center, De Boelelaan 1118, $1081 \mathrm{HZ}$ Amsterdam, The Netherlands. ${ }^{4}$ Amsterdam Rheumatology and immunology Center | Academic Medical Center, Meibergdreef 9, 1105 AZ Amsterdam, The Netherlands.

Received: 20 July 2018 Accepted: 12 November 2018

Published online: 19 December 2018

\section{References}

1. Nielen MM, van Schaardenburg D, Reesink HW, van de Stadt RJ, van der Horst-Bruinsma IE, de Koning MH, Habibuw MR, Vandenbroucke JP, Dijkmans BA. Specific autoantibodies precede the symptoms of rheumatoid arthritis: a study of serial measurements in blood donors. Arthritis Rheum. 2004;50(2):380-6.

2. Bos WH, Wolbink GJ, Boers M, Tijhuis GJ, de Vries N, van der Horst-Bruinsma IE, Tak PP, van de Stadt RJ, van der Laken CJ, Dijkmans BA, et al. Arthritis development in patients with arthralgia is strongly associated with anti-citrullinated protein antibody status: a prospective cohort study. Ann Rheum Dis. 2010;69(3):490-4.

3. Rantapaa-Dahlqvist S, de Jong BA, Berglin E, Hallmans G, Wadell G, Stenlund H, Sundin U, van Venrooij WJ. Antibodies against cyclic citrullinated peptide and IgA rheumatoid factor predict the development of rheumatoid arthritis. Arthritis Rheum. 2003;48(10):2741-9. 
4. van de Stadt LA, Witte BI, Bos WH, van Schaardenburg D. A prediction rule for the development of arthritis in seropositive arthralgia patients. Ann Rheum Dis. 2013;72(12):1920-6.

5. Nell VP, Machold KP, Eberl G, Stamm TA, Uffmann M, Smolen JS. Benefit of very early referral and very early therapy with disease-modifying antirheumatic drugs in patients with early rheumatoid arthritis. Rheumatology (Oxford). 2004;43(7):906-14

6. de Hair MJ, Landewe RB, van de Sande MG, van Schaardenburg D, van Baarsen LG, Gerlag DM, Tak PP. Smoking and overweight determine the likelihood of developing rheumatoid arthritis. Ann Rheum Dis. 2013;72(10):1654-8.

7. Sparks JA, Chen CY, Jiang X, Askling J, Hiraki LT, Malspeis S, Klareskog L, Alfredsson L, Costenbader KH, Karlson EW. Improved performance of epidemiologic and genetic risk models for rheumatoid arthritis serologic phenotypes using family history. Ann Rheum Dis. 2015;74(8):1522-9.

8. Lahiri M, Luben RN, Morgan C, Bunn DK, Marshall T, Lunt M, Verstappen SM Symmons DP, Khaw KT, Wareham N, et al. Using lifestyle factors to identify individuals at higher risk of inflammatory polyarthritis (results from the European Prospective Investigation of Cancer-Norfolk and the Norfolk Arthritis Register--the EPIC-2-NOAR Study). Ann Rheum Dis. 2014;73(1):219-26.

9. Rakieh C, Nam JL, Hunt L, Hensor EM, Das S, Bissell LA, Villeneuve E, McGonagle D, Hodgson R, Grainger A, et al. Predicting the development of clinical arthritis in anti-CCP positive individuals with non-specific musculoskeletal symptoms: a prospective observational cohort study. Ann Rheum Dis. 2015;74(9):1659-66

10. Filer A, de Pablo P, Allen G, Nightingale P, Jordan A, Jobanputra P, Bowman S, Buckley CD, Raza K. Utility of ultrasound joint counts in the prediction of rheumatoid arthritis in patients with very early synovitis. Ann Rheum Dis. 2011;70(3):500-7.

11. Lage-Hansen PR, Lindegaard H, Chrysidis S, Terslev L. The role of ultrasound in diagnosing rheumatoid arthritis, what do we know? An updated review. Rheumatol Int. 2017;37(2):179-87.

12. Mandl P, Kurucz R, Niedermayer D, Balint PV, Smolen JS. Contributions of ultrasound beyond clinical data in assessing inflammatory disease activity in rheumatoid arthritis: current insights and future prospects. Rheumatology (Oxford). 2014;53(12):2136-42.

13. Chakr RM, Mendonca JA, Brenol CV, Xavier RM, Brenol JC. Assessing rheumatoid arthritis disease activity with ultrasound. Clin Rheumatol. 2013; 32(9):1249-54

14. Colebatch AN, Edwards CJ, Ostergaard M, van der Heijde D, Balint PV, D'Agostino MA, Forslind K, Grassi W, Haavardsholm EA, Haugeberg G, et al. EULAR recommendations for the use of imaging of the joints in the clinical management of rheumatoid arthritis. Ann Rheum Dis. 2013;72(6):804-14

15. Horton SC, Tan AL, Wakefield RJ, Freeston JE, Buch MH, Emery P. Ultrasound-detectable grey scale synovitis predicts future fulfilment of the 2010 ACR/EULAR RA classification criteria in patients with new-onset undifferentiated arthritis. RMD Open. 2017;3(1):e000394

16. van de Stadt LA, Bos WH, Meursinge Reynders M, Wieringa $H$, Turkstra F, van der Laken CJ, van Schaardenburg D. The value of ultrasonography in predicting arthritis in auto-antibody positive arthralgia patients: a prospective cohort study. Arthritis Res Ther. 2010;12(3):R98.

17. Freeston JE, Wakefield RJ, Conaghan PG, Hensor EM, Stewart SP, Emery P. A diagnostic algorithm for persistence of very early inflammatory arthritis: the utility of power Doppler ultrasound when added to conventional assessment tools. Ann Rheum Dis. 2010;69(2):417-9.

18. Zufferey P, Rebell C, Benaim C, Ziswiler HR, DumusC A, So A. Ultrasound can be useful to predict an evolution towards rheumatoid arthritis in patients with inflammatory polyarthralgia without anticitrullinated antibodies. Joint Bone Spine. 2017:84(3):299-303

19. Nam JL, Hensor EM, Hunt L, Conaghan PG, Wakefield RJ, Emery P. Ultrasound findings predict progression to inflammatory arthritis in anti-CCP antibody-positive patients without clinical synovitis. Ann Rheum Dis. 2016; 75(12):2060-7.

20. Nam JL, D'Agostino MA. Role of ultrasound imaging in individuals at risk of RA. Best Pract Res Clin Rheumatol. 2017:31(1):71-9.

21. Mandl P, Naredo E, Wakefield RJ, Conaghan PG, D'Agostino MA, Force OUT. A systematic literature review analysis of ultrasound joint count and scoring systems to assess synovitis in rheumatoid arthritis according to the OMERACT filter. J Rheumatol. 2011:38(9):2055-62.

22. Szkudlarek M, Court-Payen M, Jacobsen S, Klarlund M, Thomsen HS, Ostergaard M. Interobserver agreement in ultrasonography of the finger and toe joints in rheumatoid arthritis. Arthritis Rheum. 2003;48(4):955-62.
23. D'Agostino MA, Wakefield R, Hammer HB, Vittecoq O, Galeazzi M, Balint $P$, Filippucci E, Moller I, lagnocco A, Naredo E, et al. Assessment of Omeract global power Doppler ultrasonography 44-joint scoring system and reduced joint scoring systems in rheumatoid arthritis patients treated with abatacept plus background methotrexate. Arthritis Rheum-Us. 2012;64(10):S352-3.

24. Wakefield RJ, D'Agostino MA, lagnocco A, Filippucci E, Backhaus M, Scheel AK, Joshua F, Naredo E, Schmidt WA, Grassi W, et al. The OMERACT ultrasound group: status of current activities and research directions. J Rheumatol. 2007:34(4):848-51.

25. Naredo E, Rodriguez M, Campos C, Rodriguez-Heredia JM, Medina JA, Giner E, Martinez O, Toyos FJ, Ruiz T, Ros I, et al. Validity, reproducibility, and responsiveness of a twelve-joint simplified power doppler ultrasonographic assessment of joint inflammation in rheumatoid arthritis. Arthritis Rheum. 2008; 59(4):515-22.

26. Nederlandse Vereniging voor Reumatologie (NVR): Richtlijn Diagnostiek van Reumatoïde Artritis. Wat is de waarde van echografie voor de diagnose Reumatoïde Artritis. 2016;38-47. https://www.nvr.nl/wp-content/uploads/ 2018/09/Richtlijndiagnostiekreumatoafdeartritis_FINAL-def.pdf. Accessed 25 Dec 2018.

27. Scheel AK, Hermann KG, Kahler E, Pasewaldt D, Fritz J, Hamm B, Brunner E, Muller GA, Burmester GR, Backhaus M. A novel ultrasonographic synovitis scoring system suitable for analyzing finger joint inflammation in rheumatoid arthritis. Arthritis Rheum. 2005;52(3):733-43.

28. Twisk JWR. Applied longitudinal data analysis for epidemiology: a practical guide. Cambridge: Cambridge University Press; 2013

29. Vyas S, Bhalla AS, Ranjan P, Kumar S, Kumar U, Gupta AK. Rheumatoid arthritis revisited - advanced imaging review. Pol J Radiol. 2016;81:629-35.

30. Ikeda K, Seto Y, Ohno S, Sakamoto F, Henmi M, Fukae J, Narita A, Nakagomi $D$, Nakajima $H$, Tanimura $K$, et al. Analysis of the factors which influence the measurement of synovial power Doppler signals with semi-quantitative and quantitative measures - a pilot multicenter exercise in Japan. Mod Rheumatol. 2014;24(3):419-25.

31. D'Agostino MA, Terslev L, Aegerter P, Backhaus M, Balint P, Bruyn GA Filippucci E, Grassi W, lagnocco A, Jousse-Joulin S, et al. Scoring ultrasound synovitis in rheumatoid arthritis: a EULAR-OMERACT ultrasound taskforcePart 1: definition and development of a standardised, consensus-based scoring system. RMD open. 2017:3(1):e000428.

32. van der Ven M, van der Veer-Meerkerk M, Ten Cate DF, Rasappu N, Kok MR, Csakvari D, JMW H, Gerards AH, Luime JJ. Absence of ultrasound inflammation in patients presenting with arthralgia rules out the development of arthritis. Arthritis Res Ther. 2017;19(1):202.

33. Sahbudin I, Pickup L, Nightingale P, Allen G, Cader Z, Singh R, de Pablo P, Buckley CD, Raza K, Filer A. The role of ultrasound-defined tenosynovitis and synovitis in the prediction of rheumatoid arthritis development. Rheumatology (Oxford). 2018. https://doi.org/10.1093/rheumatology/key025. [Epub ahead of print].

Ready to submit your research? Choose BMC and benefit from

- fast, convenient online submission

- thorough peer review by experienced researchers in your field

- rapid publication on acceptance

- support for research data, including large and complex data types

- gold Open Access which fosters wider collaboration and increased citations

- maximum visibility for your research: over $100 \mathrm{M}$ website views per year

At BMC, research is always in progress.

Learn more biomedcentral.com/submissions 